\title{
Estilos de aprendizaje y rendimiento académico en estudiantes universitarios de Psicología en Talca
}

\author{
Learning styles and academic performance in university students of \\ Psychology in Talca
}

\author{
FABIOLA DEL CARMEN CARRASCO CIFUENTES \\ Licenciada en Psicología, Universidad Autónoma de Chile \\ Ilustre Municipalidad de Maule, Departamento de Educación \\ fabiolacarrasco.psicologa@gmail.com

\section{MARIELA ALEJANDRA GONZÁLEZ MÉNDEZ} \\ Licenciada en Psicología, Universidad Autónoma de Chile \\ Fondo Esperanza, Constitución, Chile \\ mariela.gm84@gmail.com
}

\section{RESUMEN}

El concepto de los estilos de aprendizaje resulta interesante porque ofrece una teoría respaldada en sugerencias y aplicaciones prácticas con posibilidades de lograr aprendizajes mucho más efectivos. El presente estudio tiene como objetivo evaluar los estilos de aprendizaje que presentan los estudiantes de Psicología de la Universidad Autónoma de Chile, sede Talca, según su rendimiento académico y año de ingreso a la universidad. El estudio es cuantitativo de diseño transversal. La muestra estuvo constituida por 164 estudiantes, con años de ingreso 2004, 2005, 2006 y 2007, de los cuales 113 corresponden a mujeres y 51 a hombres. Se utilizó el cuestionario de Honey-Alonso de Estilos de Aprendizaje (CHAEA), con adaptaciones para el contexto local chileno. El rendimiento académico, expresado en el promedio acumulado, fue tomado de los archivos de la carrera de Psicología de la Universidad Autónoma de Chile, sede Talca. La evaluación de los puntajes obtenidos por los alumnos en los estilos de aprendizaje fueron relacionados con el rendimiento académico, el año de ingreso y el género de los estudiantes. Los resultados muestran que los estudiantes no poseen un estilo de aprendizaje característico, sino que presentan una preferencia moderada hacia cada uno de los estilos de aprendizaje. En conclusión, no se presenta evidencia que permita establecer que un solo estilo de aprendizaje podría ser una herramienta docente útil para generar metodologías apropiadas para conseguir un mejor rendimiento académico.

Palabras clave: estilos de aprendizaje, rendimiento académico, psicología, estudiantes universitarios. 


\section{ABSTRACT}

The concept of learning styles is interesting because it offers a theory based on suggestions and practical applications with the potential to achieve a more effective way of learning. The objective of the present study is to evaluate the learning styles presented by Psychology students of the Universidad Autónoma de Chile, Talca campus, according to their academic performance and admission year. The study is a quantitative cross-sectional design. The samples were constructed of 164 students, admitted in 2004, 2005, 2006 and 2007, of which 113 were women and 51 men. The Honey-Alonso Learning Styles (CHAEA) questionnaire was used, with adaptions for the local Chilean system. The academic performance expressed in the cumulative average, was obtained from the corresponding files of a university Psychology course in Talca. The evaluation of the scores obtained by students in learning styles related to the academic performance, the year of admission, and student's gender. The results showed that students do not have a distinctive learning style, but present a moderate preference towards each type of learning style. In conclusion, there is no evidence to establish that a single learning style could be a useful teaching tool to generate appropriate methodologies to achieve better academic performance.

Key words: learning style, academic performance, psychology, university students.

\section{INTRODUCCIÓN}

La noción de estilos de aprendizaje tiene sus antecedentes en el campo de la psicología cognitiva. Este concepto fue utilizado por primera vez en los años 50 del siglo pasado por los llamados "psicólogos cognitivistas", quienes comenzaban a prestar especial atención al hombre desde el punto de vista de la cognición, incentivados por el desarrollo de la lingüística y por la incipiente revolución tecnológica en los campos de la informática y las comunicaciones, a partir del surgimiento de las computadoras, además de los descubrimientos en las ciencias neurológicas y el debilitamiento del conductismo (Cabrera \& Fariñas, 2005).

Entre los investigadores cognitivos, Witkin fue uno de los primeros que se interesó por el estudio de los "estilos de aprendizaje", entendiéndolo como expresión de las formas particulares de los individuos de percibir y procesar la información (Woolfolk, 2015).

Con el tiempo, algunos psicólogos de la educación, a diferencia de los teóricos de la personalidad, en lugar de "estilo cognitivo" han preferido el uso 
del término "estilo de aprendizaje", por considerar que refleja mejor el carácter multidimensional del proceso de adquisición de conocimientos en el contexto escolar (Sandín, 2003).

Sin embargo, para Willing (1988, citado en Navarro, 2001) la noción de estilo de aprendizaje se superpone a la de estilo cognitivo, al ser más comprensiva, puesto que incluye comportamientos cognitivos y afectivos que indican las características y las maneras de percibir, interactuar y responder al contexto de aprendizaje por parte del aprendiz.

Todo lo anterior derivó en una amplia diversidad de definiciones, clasificaciones e instrumentos de diagnósticos, conformados por los más disímiles enfoques y modelos teóricos con respecto a este objeto de estudio (Cabrera \& Fariñas, 2005).

Al respecto, se han desarrollado distintos modelos y teorías sobre estilos de aprendizaje, los cuales ofrecen un marco conceptual que permite entender los comportamientos diarios en el aula y cómo se relacionan con la forma en la que están aprendiendo los alumnos, además del tipo de acción que puede resultar más eficaz en un momento dado (Gómez, Aduna, García, Cisneros \& Padilla, 2004).

A nivel general, existe gran diversidad de concepciones teóricas que han tocado, ya sea de forma explícita o implícita, los diferentes estilos de aprendizaje. Cada una de ellas posee su atractivo, por lo que es posible tomar una u otra dependiendo de los aspectos del proceso de aprendizaje que sean de interés.

La categorización de los estilos de aprendizaje de la presente investigación tiene como base dos modelos o concepciones teóricas de suma importancia, los que serán abordados a continuación:

a) Modelo Experiencial de Kolb (1984): Este modelo trata de explicar cómo aprendemos, es decir, cómo asimilamos la información, solucionamos problemas y tomamos decisiones.

Thompson y Aveleyra (2003) mencionan que Kolb fundamentó su concepción del aprendizaje experiencial teniendo como puntos clave de esta teoría que el aprendizaje: 
- Debe ser concebido como un proceso, y no simplemente en términos de resultados.

- Debe ser construido de modo continuo sobre la experiencia, que requiere la solución de conflictos entre modos dialécticos de adaptarse a la realidad.

- Debe ser holístico, global, de adaptación al mundo con transacciones entre la persona y el medio.

- Contar con creación de conocimiento.

Este modelo describe la adaptación al medio físico y social y consta de cuatro etapas que constituyen un ciclo completo, tal como se señala a continuación (Delgado, 2004):

$1^{\circ}$ Inicio del aprendizaje a partir de la experiencia inmediata y concreta.

$2^{\circ}$ Desarrollo de la observación y reflexión acerca de los hechos que ocurren como resultado de la experiencia concreta.

$3^{\circ}$ A partir de la observación y la reflexión, se forman los conceptos, abstracciones y generalizaciones, las cuales pueden ser asimiladas a una teoría; de ellas, es factible deducir nuevas implicaciones para poder realizar una acción más eficaz.

$4^{\circ}$ Finalmente, el establecimiento de deducciones que pueden ser puestas a prueba en nuevas situaciones, con lo cual se puede continuar con el proceso de aprendizaje.

Según Kolb, el aprendizaje experiencial funciona de los cuatro modos, lo que requiere cuatro tipos de competencias: la experiencia concreta es propia de personas que se sumergen en la inmediatez del momento, basándose en sus respuestas intuitivas y afectivas a las situaciones. Complementariamente, la conceptualización abstracta se refiere al pensamiento lógico y la evaluación racional para crear ideas que integren sus observaciones en teorías firmes y fundamentadas. La observación reflexiva asume una perspectiva tentativa, imparcial hacia la situación de aprendizaje (una especie de voluntad de considerar pacientemente numerosas alternativas). Finalmente, la experimentación activa acentúa la acción, la participación, y el riesgo al aprender, con un cierto énfasis 
en generar conceptos previamente probados empíricamente (Rodríguez, 2003).

Este modelo indica que para tener un aprendizaje eficaz son necesarios los cuatro aspectos, diametralmente opuestos, pudiendo elegir la persona que aprende entre estas competencias de aprendizaje para aplicarlas a situaciones concretas. Esto, lleva a poder identificar la existencia de dos dimensiones básicas que subyacen a esta concepción del proceso de aprendizaje: la dimensión concreta-abstracta y la dimensión activa-reflexiva (Delgado, 2004).

Producto de la interacción entre las dos dimensiones del aprendizaje, Kolb (1984, citado en Ubeda \& Escribano, 2002) reconoce cuatro estilos de aprendizaje; estos, están presente en su cuestionario "Learning Style Inventory" - LSI:

- Divergente: son individuos con habilidad imaginativa, que ven situaciones desde diferentes perspectivas. Son emotivos y buenos generando ideas.

- Asimilador: personas con habilidad para crear modelos teóricos, con razonamiento inductivo; se preocupan más por los conceptos que por el uso práctico de las teorías.

- Convergente: son personas interesadas en la aplicación práctica de las ideas; resultan buenas en situaciones donde hay más de una respuesta. No son emotivos.

- Acomodador: son personas con habilidad para llevar a cabo planes orientados a la acción. También son: arriesgados, les gustan las nuevas experiencias, se adaptan a las circunstancias inmediatas, son intuitivos y aprenden por tanteo y error.

Es interesante resaltar que, los estilos de aprendizaje según la mirada de Kolb son situacionales, o sea, el sujeto puede utilizar hoy uno u otro estilo, pero puede que mañana no se sirva del mismo (Rodríguez, 2003).

b) Modelo de Honey y Mumford (1992): estos autores partieron de una reflexión académica y de un análisis de la teoría de Kolb para llegar a una aplicación de los estilos de aprendizaje en la formación de directivos del Reino Unido.

A los autores les interesó averiguar por qué en una situación en la que dos personas comparten texto y contexto una de ella aprende y otra no. Una explicación 
está en que los estilos de aprendizaje de cada persona originan diferentes respuestas y comportamientos ante el aprendizaje (Thompson \& Aveleyra, 2003). Honey y Mumford (1992, citado en Alonso, Gallego \& Honey, 2002) asumen gran parte de las teorías de Kolb, insisten en el proceso circular del aprendizaje en cuatro etapas y sostienen la importancia del aprendizaje por la experiencia. En cambio, no les parece adecuado el instrumento para detectar los estilos de aprendizaje que este autor utiliza, ni sus descripciones de los estilos de aprendizaje para el grupo en concreto con el que trabajan.

Las diferencias de este modelo con el modelo de Kolb se pueden concretar en tres puntos fundamentales (Alonso, Gallegos \& Honey, 2002):

- Las descripciones de los estilos son más detalladas y se basan en la acción de los sujetos.

- Las respuestas al cuestionario son un punto de partida y no un final. Existe un punto de arranque y un diagnostico, seguido de un tratamiento de mejora. Se trata de facilitar una guía práctica que ayude y oriente al individuo en su mejora personal y también en la de sus colegas y subordinados.

- Describen un cuestionario con ochenta ítems, que permiten analizar una mayor cantidad de variables que el test propuesto por Kolb.

Según Honey (1986, citado en Thompson \& Aveleyra, 2003), lo ideal sería que todo el mundo fuera capaz de experimentar, reflexionar, elaborar hipótesis y aplicar. Pero, lo cierto es que, las personas son más capaces de una cosa que de otra. Los estilos de aprendizaje son, entonces, la interiorización por parte de cada sujeto de una etapa determinada del ciclo.

Al igual que en el modelo de Kolb, para Honey y Mumford los estilos de aprendizaje son cuatro, que coinciden, a su vez, con las cuatro fases de un proceso cíclico de aprendizaje (Alonso, Gallego \& Honey, 2002):

- Estilo Activo: las personas que tienen predominancia del estilo activo se implican plenamente y sin prejuicios en nuevas experiencias. Son de mente abierta, nada escépticos y acometen con entusiasmo las tareas nuevas; se centran en el aquí y el ahora y les encanta vivir nuevas experiencias. Sus días están llenos de actividad, por ello, tan pronto como desciende la excitación de una tarea, comienzan a buscar la próxima. Crecen ante los 
desafíos que suponen nuevas experiencias, y se aburren con los plazos largos. Además, son individuos de grupos, que se involucran en los asuntos de los demás y centran a su alrededor todas las actividades.

- Estilo Reflexivo: a los reflexivos les gusta considerar las experiencias y observarlas desde distintas perspectivas. Recogen datos y los analizan detalladamente antes de llegar a una conclusión. Son prudentes y analizan todas las implicaciones de cualquier acción antes de ponerse en movimiento; disfrutan observando la actuación de los demás, los escuchan y no intervienen hasta que se han adueñado de la situación. También, crean a su alrededor un aire ligeramente distante y condescendiente.

- Estilo Teórico: los teóricos adaptan e integran las observaciones dentro de teorías lógicas y complejas. Enfocan los problemas de forma escalonada, por etapas lógicas. Les gusta analizar y sintetizar. Son profundos en su forma de pensamiento al momento de establecer principios, teorías y modelos. Además, buscan la racionalidad y la objetividad, huyendo de lo subjetivo y de lo ambiguo.

- Estilo Pragmático: el punto fuerte de las personas con predominancia en este estilo es la aplicación práctica de las ideas. Descubren el aspecto positivo de las nuevas ideas y aprovechan la primera oportunidad para experimentarla. Les gusta actuar rápidamente y con seguridad con aquellas ideas y proyectos que les atraen. Asimismo, tienden a ser impacientes cuando hay personas que teorizan.

El instrumento que utilizan estos autores para detectar los distintos estilos de aprendizaje es el Learning Styles Questionnaire (L.S.Q), compuesto por ochenta ítems. En él, el sujeto debe responder si está de acuerdo o en desacuerdo con las preguntas, que son, en su mayoría, comportamentales, es decir, describen una acción que alguien puede realizar. EI LSQ está diseñado para detectar las tendencias generales del comportamiento personal (Alonso, Gallego \& Honey, 2002).

Alonso, Gallego y Honey (2002), basándose en la descripción de los estilos de aprendizaje según la conceptualización de Honey y Mumford, añadieron una lista de características, las cuales creen que determinan con claridad el campo de destrezas de cada uno de los cuatro estilos. 
Las principales características del estilo activo, en orden de prioridad son: animador, improvisador, descubridor, arriesgado, espontáneo, creativo, aventurero, inventor, generador de ideas, innovador, líder, competitivo, entre otras.

Las personas que tengan un predominio claro del estilo reflexivo tienen como principales características las siguientes: el ser ponderado, concienzudo, receptivo, analítico, exhaustivo, observador, paciente, detallista, previsor de alternativas, investigador, asimilador, lento, prudente, entre otras.

Por otro lado, el estilo teórico se caracteriza principalmente por ser metódico, lógico, objetivo, crítico y estructurado. Entre las características secundarias se encuentran: el ser disciplinado, planificado, sistemático, sintético, razonador, perfeccionista y buscar hipótesis.

Por último, las personas en las que predomina un estilo pragmático se caracterizan por ser experimentadores, prácticos, directos, eficaces y realistas. También, suelen ser técnicos, rápidos, decididos, planificadores, objetivos, concretos, seguros de sí, entre otras.

Por otro lado, algunos estudios como el de López \& Ballesteros (2003) identificaron las preferencias personales en cuanto al estilo de aprendizaje en estudiantes de Enfermería. Para ello, utilizaron el cuestionario de Honey-Alonso de estilos de aprendizaje (CHAEA), baremado para la población española de estudiantes de Enfermería, el cual se administró a una muestra de 102 individuos de una misma escuela. Los resultados obtenidos se asemejan a los presentados por los autores de la versión española del cuestionario: los alumnos prefieren un estilo de aprendizaje reflexivo, aunque se muestran más teóricos y activos que en el citado estudio. Con respecto al curso, entre primero y segundo no existen diferencias significativas en ninguno de los estilos de aprendizaje, como tampoco las hay entre primero y tercero. Sí se pueden observar diferencias entre los estudiantes de segundo y tercero.

Martínez (2003) realizó una investigación con alumnos de la carrera de Administración de Hoteles y Restaurantes y con profesionales egresados de escuelas relacionadas con la hotelería que trabajan en la ciudad de Puebla, México. Para ello, utilizó el inventario de estilos de aprendizaje de Kolb. En el estudio se llegó a la conclusión de que no existe diferencia entre los estilos de aprendizaje de los estudiantes y los profesionales ya egresados. Esto se da porque, posiblemente, 
las personas que cursan carreras relacionadas con hotelería, así como las que trabajan en esta industria, quizás, enfrenten retos similares y posean el mismo tipo de habilidades, por lo que tienen el mismo estilo de aprendizaje.

Por su parte, Ruiz, Trillos \& Morales (2006) exploraron los estilos de aprendizaje de estudiantes de segundo semestre de los programas académicos de la Universidad Tecnológica de Bolívar y su posible relación con el rendimiento académico. Para esta investigación se aplicó el CHAEA a una población de 101 estudiantes. Los resultados indican un mayor nivel de preferencia por los estilos activo, teórico, pragmático y una menor preferencia por el estilo reflexivo, encontrándose una correlación positiva entre el estilo teórico y el rendimiento académico.

En Chile, Romo, López, Tovar \& López (2004) realizaron una investigación cuyo fin fue determinar cambios en los estilos de aprendizaje de la promoción 2000 de la carrera de Nutrición y Dietética durante un período de dos años, además trataron de establecer la relación de los estilos con el rendimiento académico. Al respecto, se utilizó el Inventario de Estrategias de Aprendizaje de Schmeck, adaptado para Chile por Trufello. Los resultados indicaron que los estudiantes no modificaron los estilos de aprendizaje, a pesar de haber avanzado en el proceso educativo y no obtuvieron mejores notas, y al haberlas, este cambio no resultó estadísticamente significativo.

Von Chrismar (2005) realizó un estudio acerca de los estilos de aprendizaje de los estudiantes de la Universidad Austral de Chile, mediante la aplicación del cuestionario CHAEA, con el que se identificaron los perfiles de aprendizaje grupales para cinco carreras (Bioquímica, Ingeniería Comercial, Tecnología Médica, Química y Farmacia y Medicina Veterinaria). Los resultados indicaron un predominio claro del estilo reflexivo (59\%), seguido del estilo teórico (17\%), luego del pragmático (14\%) y, por último, del estilo activo (10\%).

Mientrasque, Saavedra\& Reynaldos (2006) investigaron el perfil de personalidad y rendimiento académico en una muestra de 287 estudiantes universitarios. Para ello, se utilizaron tres test psicológicos: el Inventario de Preferencias Personales de Allen Edwards, el Cuestionario de Estilos Vocacionales de John Holland y el Cuestionario de Estilos y Estrategias del Aprendizaje de David Kolb. Los resultados indican que las características de personalidad predominantes de los alumnos, según el test de Edwards, son la persistencia y el exhibicionismo; de acuerdo con el test de Kolb, se encontraron alumnos que, lejos de estar abiertos a metodologías 
flexibles y dinámicas, parecen aprender mejor con estilos más pasivos y teóricos. En general, los alumnos se encuentran bien ubicados en las carreras que han elegido, según lo encontrado por el test de Holland, excepto en Ciencias Agrarias y Forestales, carreras adonde parecen llegar sin una clara vocación.

Cabe destacar que, el presente estudio tiene como objetivo evaluar los estilos de aprendizaje que presentan los estudiantes de Psicología de una universidad chilena en Talca, según rendimiento académico y año de ingreso a la universidad. La relevancia de esta investigación radica en que permitirá tener una descripción de los estilos de aprendizaje que los alumnos utilizan en el proceso de formación universitaria. Al respecto, la población de estudio no ha sido motivo de intervención alguna por parte de esta área investigativa, por lo que el conocer la forma en la que se expresan los estilos de aprendizaje en los grupos de estudiantes universitarios, en este caso en la carrera de Psicología, lograría ser un instrumento docente muy útil para crear metodologías apropiadas, adecuando el estilo de enseñanza de los docentes, con el objetivo de conseguir un mejor rendimiento académico y, a la vez, permitiría bosquejar métodos de evaluación más idóneos para evidenciar el progreso de los estudiantes.

\section{MÉTODO}

Esta investigación corresponde a un estudio cuantitativo, de diseño descriptivo. De una población de 289 estudiantes de Psicología (de primero a quinto año) de la Universidad Autónoma de Chile, sede Talca, se seleccionó una muestra conformada por 164 alumnos, lo que corresponde al $56,74 \%$ del total. La muestra estuvo constituida por estudiantes con años de ingreso 2004, 2005, 2006 y 2007, quienes participaron voluntariamente, tanto en la aplicación en sala como en el llamado publicado; de ellos, 113 fueron mujeres, lo que equivale al 68,9\%, y 51 eran hombres, lo que equivale al $31,1 \%$.

Las variables analizadas fueron las siguientes:

- Estilos de aprendizaje: estos se subdividen en cuatro estilos (activo, reflexivo, teórico y pragmático), los cuales fueron medidos a través del Cuestionario de Estilos de Aprendizaje de Honey-Alonso (CHAEA). Cabe mencionar que, se puede presentar más de un estilo de aprendizaje predominante por individuo, lo que no implica que los otros estilos no sean utilizados por esta misma persona. 
- Rendimientoacadémico:correspondeal promedioacumulado hasta el primer semestre del año académico 2007. Este fue solicitado al Departamento de Psicología. El rendimiento abarca una escala comprendida entre: 1,0 a 7,0, donde: 7,0 a 6,0 corresponde a muy bueno (MB); 5,9 a 5,0 se define como bueno (B); 4,9 a 4,0 suficiente (S); y, de 3,9 a 1,0 insuficiente (I). La importancia de esta variable radica en que permitirá conocer si los alumnos presentan un estilo de aprendizaje en particular, según las categorías antes señaladas.

- Año de ingreso: son los ingresos académicos de la carrera de Psicología 2004, 2005, 2006 y 2007. Es importante destacar que, el ingreso 2003 no fue considerado para esta investigación, pues correspondía al grupo de alumnos en proceso de práctica profesional y en desarrollo de su tesis de grado. Esta variable se solicitó, junto a las variables demográficas, dentro del Cuestionario de Estilos de Aprendizajes (CHAEA).

- Género: esta variable se incluyó en la investigación para tener una descripción más acabada de los estilos de aprendizaje de la muestra. Esta variable fue solicitada dentro del Cuestionario de Estilos de Aprendizajes (CHAEA).

De una población de 289 estudiantes de Psicología de primero a quinto año, se trabajó con una muestra de 164 alumnos, lo que corresponde al $56,74 \%$ del total. La muestra estuvo constituida por alumnos con años de ingreso 2004, 2005, 2006 y 2007 que participaron voluntariamente, tanto en la aplicación en sala como en el llamado publicado. De ese grupo, 113 fueron mujeres lo que equivale al $68,9 \%$ y 51 hombres que constituyen el $31,1 \%$.

Con respecto a la división por año de ingreso a la universidad, se puede indicar que el mayor porcentaje de alumnos se encuentra en el año 2007 que corresponde al $43,3 \%(n=71)$ de la muestra total. Por el contrario, el menor porcentaje de alumnos se ubica en el año de ingreso 2004 que equivale al 17,7\% $(n=29)$. El ingreso 2006 corresponde a 32 estudiantes $(19,5 \%$ ) y el ingreso 2005 es de 32 estudiantes (19,5\%).

Para determinar los estilos de aprendizaje de los estudiantes de Psicología con años de ingreso 2004, 2005, 2006 y 2007 de la Universidad Autónoma de Chile, sede Talca, se aplicó el Cuestionario Honey-Alonso de Estilos de Aprendizaje (CHAEA). Este instrumento consta de 80 ítems breves y dicotómicos, distribuidos 
aleatoriamente. A partir de esos ítems se evalúan los cuatro estilos de aprendizaje. Los 80 ítems se estructuran en cuatro grupos de 20 preguntas correspondientes a cada uno de los estilos de aprendizaje. La puntación es sumativa para cada uno de los grupos, indicando el nivel que posee la persona en cada estilo. Las respuestas a los 80 ítems del cuestionario son de dos posibles respuestas: positiva (+) (cuando se está más de acuerdo que en desacuerdo con la aseveración) y negativa (-), (al estar en desacuerdo con la aseveración). Los ítems deben responderse en su totalidad con esas opciones de respuesta.

El CHAEA está conformado por los siguientes elementos:

- Hoja de instrucciones: se detalla lo que se mide y la forma de responder el inventario. También, se solicitan datos relevantes del sujeto evaluado.

- Instrumento de aplicación: está conformado por 80 ítems de preguntas 0 aseveraciones, con un espacio adicional al costado izquierdo de cada una de ellas, a fin de que el sujeto asigne el signo con el que se identifique más.

- Hoja de perfil de aprendizaje: se adjudica el puntaje obtenido en cada estilo, a fin de ser distribuido posteriormente en un gráfico de frecuencias.

Es importante resaltar que, previo a las aplicaciones del instrumento, se solicitó al Departamento de Psicología una lista con los alumnos matriculados en el año 2007, con la finalidad de conocer la población total de la carrera y extraer la muestra a trabajar, es decir, los alumnos cuyos años de ingreso corresponden a los períodos 2004, 2005, 2006 y 2007. Al respecto, quedaron fuera de la muestra los alumnos que ingresaron en el 2003, pues estos se encontraban en práctica profesional.

La aplicación del cuestionario se realizó a principios del segundo semestre del año 2007. La primera instancia de aplicación fue a los alumnos presentes durante las clases de gran convocatoria. Allí, se aplicaron un total de 134 cuestionarios. Luego, se procedió a hacer una lista de todos los alumnos que no habían contestado el instrumento para captarlos en una segunda aplicación. En este punto, se encontró que la cantidad de cuestionarios que faltaba por aplicar era excesiva en contraste con la realidad percibida en terreno. Dado lo anterior, se solicitó nuevamente un listado de los alumnos, tomando como filtro la toma de carga académica correspondiente al segundo semestre del año 2007, 
y se descartaron a los alumnos que, por una u otra razón, ya no se encontraban vigentes en la institución académica.

Al contar con la lista de alumnos con carga académica al día, se procedió, en primera instancia, a anular los cuestionarios contestados en la aplicación en sala, cuyos alumnos no tomaron ramos en la fecha estipulada por la universidad. También, se extrajeron dos cuestionarios que pertenecían a alumnas convalidadas al actual semestre académico, pues no tenían notas en el registro de la universidad. De esa forma, hubo un total de 125 cuestionarios válidos. Posteriormente, se realizó el llamado de captación a los alumnos restantes, el cual estuvo constituido por un total de 75 alumnos; de ellos, asistieron 39 sujetos.

En ambas aplicaciones, previo a la entrega del cuestionario, se informó a los individuos que los rasgos que son motivo del estudio pretendían cuantificar características en las que no había respuestas correctas o incorrectas. Además, se dejó en claro que el instrumento aplicado medía atributos relacionados con la disposición personal del individuo frente al aprendizaje.

Las instrucciones para la realización del cuestionario se entregaron verbalmente, como también de forma escrita. Estas fueron breves y claras y se relacionaron únicamente con los procedimientos de respuesta. No se aclararon ítems particulares.

Se solicitó completar aspectos como sexo, edad, RUT y año de ingreso a la universidad dentro del cuestionario, pues eran datos necesarios para asignar a los estudiantes a los distintos años de ingreso; además, se pidió el promedio de cada uno de los alumnos a la Escuela de Psicología.

Una vez completada la aplicación del instrumento, se procedió de la siguiente manera:

- Se adjudicó una hoja de perfil de aprendizaje a cada uno de los cuestionarios.

- Se marcó con un círculo en la hoja de perfil cada uno de items a los que el estudiante otorgó un signo más (+).

- Se sumó el número total de círculos existentes en cada columna. 
- Se colocaron los totales en el sector señalado, para luego distribuirlos en el gráfico de frecuencias.

- Se solicitó a la universidad los promedios de todos los sujetos de la muestra.

- Se ingresaron los datos personales, resultados obtenidos y promedios de notas de la muestra a la plantilla en Excel.

- Se traspasaron los datos desde la plantilla Excel al programa computacional Statistical Product and Service Solutions (SPSS) para el análisis.

Para la interpretación y tabulación del cuestionario CHAEA, se hizo uso de la normalización existente en español, la cual permite interpretar y obtener datos, tales como el porcentaje de preferencia que tiene el alumno con respecto al estilo de aprendizaje que lo identifique.

A nivel descriptivo se emplearon: medidas de frecuencia, porcentajes, promedios, mínimas, máximas y desviación estándar. Esto permitió caracterizar a la población objeto de estudio en cada una de las variables relevantes. Para la realización de un análisis más complejo, se empleó la correlación de Pearson y Chi cuadrado para establecer las relaciones entre las variables existentes. Adicionalmente, se empleó el procedimiento de análisis de varianza (ANOVA). Para todos los cálculos mencionados se utilizó el paquete estadístico Statistical Product and Service Solutions (SPSS, versión 15.0).

\section{RESULTADOS}

El perfil de aprendizaje de la muestra estudiada, de acuerdo con la puntuación obtenida en cada estilo, evidencia que las medias corresponden al nivel de preferencia moderada (Tabla 1), de acuerdo a los baremos formulados por Alonso, Gallego \& Honey (2002).

Al realizar una comparación del perfil de aprendizaje de la muestra, con las medias obtenidas en las investigaciones de Alonso con universitarios de Madrid (Alonso et al., 2002), se encuentra que los estudiantes presentaron una mayor puntuación en los estilos activo, teórico y pragmático. 
Por otro lado, la media del rendimiento académico de los alumnos se encuentra dentro del rango suficiente, siendo el promedio mínimo un 2,9 y el máximo un 6,4 como se puede apreciar en la Tabla 1.

\section{Tabla 1}

Estadísticos descriptivos del rendimiento académico y estilos de aprendizaje de los estudiantes de Psicología.

\begin{tabular}{lcccc}
\hline & Mínimo & Máximo & Media & Desv. típ. \\
\hline Rendimiento académico & 29 & 64 & 48,49 & 6,204 \\
\hline Estilo activo & 3 & 18 & 11,53 & 3,079 \\
\hline Estilo reflexivo & 7 & 20 & 14,93 & 2,775 \\
\hline Estilo teórico & 5 & 18 & 12,74 & 3,025 \\
\hline Estilo pragmático & 6 & 18 & 12,21 & 2,636 \\
\hline
\end{tabular}

Por otro lado, se puede indicar que un $50 \%$ de los estudiantes de la carrera de Psicología presenta un rendimiento académico bueno, aunque también hay que considerar un porcentaje moderado con rendimiento suficiente (37,2\%). Un $11 \%$ posee un rendimiento insuficiente y un 1,8\% muy bueno.

Con respecto a los resultados en cada estilo de aprendizaje, se puede apreciar en primera instancia que dentro del estilo activo el mayor porcentaje $(43,3 \%)$ se ubica en el rango preferencia moderada, lo que indica que gran parte de los estudiantes presentan una preferencia moderada hacia ese estilo, mientras que, el porcentaje más bajo $(5,5 \%)$ se concentra en el rango de preferencia muy baja. Es importante destacar que, la preferencia muy alta representa el $22,6 \%$ del porcentaje total, la preferencia baja es de $12,8 \%$ y a la preferencia alta corresponde un $15,9 \%$.

Dentro del estilo reflexivo, el mayor porcentaje de estudiantes $53,7 \%$ se ubica en el rango de preferencia moderada; 18,9\% preferencia baja, 8,5\% preferencia muy baja, $18,3 \%$ preferencia alta, mientras que el porcentaje menor $0,6 \%$ está agrupado en el rango de preferencia muy alta.

Al hacer referencia al estilo teórico, se observa que el mayor porcentaje (46,3\%) se encuentra en el rango preferencia moderada, al igual que en los dos estilos de aprendizaje anteriormente expuestos. Esto quiere decir que, gran parte de los alumnos presentan una preferencia moderada hacia este estilo. Mientras que, el 
menor porcentaje de estudiantes (3\%) se agrupa en el rango preferencia muy baja. Se debe destacar que, en segundo lugar se encuentra la preferencia muy alta con un $20,7 \%$ del total; mientras que con un $18,9 \%$ se ubica la preferencia alta y con un $11 \%$ la preferencia baja.

Por último, al referirse al estilo pragmático se aprecia que el mayor porcentaje $(39,6 \%)$ se posiciona en el rango preferencia moderada, es decir, que una importante cantidad de estudiantes posee una preferencia moderada hacia el estilo en cuestión. Este resultado sella una igualdad, a nivel de rango de preferencia, entre los cuatro estilos de aprendizaje. El menor porcentaje de alumnos dentro del estilo pragmático $(6,1 \%)$ se concentra en el rango preferencia muy baja; la preferencia baja corresponde al 22,6\%, moderada al 39,6\%, alta $20,7 \%$, y preferencia muy alta obtuvo $11,0 \%$ del total, quedando dentro de los porcentajes más bajos en este estilo.

Por otro lado, se estimó la relación entre los estilos de aprendizaje y el rendimiento académico (Tabla 2), observándose que a un nivel de $p<0,05$ de significancia, el único estilo que se correlaciona con el rendimiento académico es el estilo activo, siendo una correlación negativa. Esto significa que, a mayor rendimiento académico, existe un menor puntaje en el estilo activo en los estudiantes. 
Tabla 2

Correlación entre estilos de aprendizaje y rendimiento académico.

\begin{tabular}{|c|c|c|c|c|c|c|}
\hline & & Rendimiento & Activo & Reflexivo & Teórico & Pragmático \\
\hline \multirow[t]{3}{*}{ Rendimiento } & $\begin{array}{l}\text { Correlación de } \\
\text { Pearson }\end{array}$ & 1 & $-0,163^{*}$ & 0,133 & 0,128 & $-0,013$ \\
\hline & Sig. (bilateral) & & 0,038 & 0,089 & 0,102 & 0,872 \\
\hline & $\mathrm{N}$ & 164 & 164 & 164 & 164 & 164 \\
\hline \multirow[t]{3}{*}{ Activo } & $\begin{array}{l}\text { Correlación de } \\
\text { Pearson }\end{array}$ & $-0,163^{*}$ & 1 & $-0,258^{\star *}$ & $-0,246^{\star *}$ & 0,097 \\
\hline & Sig. (bilateral) & 0,038 & & 0,001 & 0,002 & 0,214 \\
\hline & $\mathrm{N}$ & 164 & 164 & 164 & 164 & 164 \\
\hline \multirow[t]{3}{*}{ Reflexivo } & $\begin{array}{l}\text { Correlación de } \\
\text { Pearson }\end{array}$ & 0,133 & $-0,258^{\star *}$ & 1 & $0,546^{\star *}$ & $0,322^{\star *}$ \\
\hline & Sig. (bilateral) & 0,089 & 0,001 & & 0 & 0 \\
\hline & $\mathrm{N}$ & 164 & 164 & 164 & 164 & 164 \\
\hline \multirow[t]{3}{*}{ Teórico } & $\begin{array}{l}\text { Correlación de } \\
\text { Pearson }\end{array}$ & 0,128 & $-0,246^{\star *}$ & $0,546^{* *}$ & 1 & $0,404^{\star *}$ \\
\hline & Sig. (bilateral) & 0,102 & 0,002 & 0 & & 0 \\
\hline & $\mathrm{N}$ & 164 & 164 & 164 & 164 & 164 \\
\hline \multirow[t]{3}{*}{ Pragmático } & $\begin{array}{l}\text { Correlación de } \\
\text { Pearson }\end{array}$ & $-0,013$ & 0,097 & $0,322^{\star *}$ & $0,404^{\star *}$ & 1 \\
\hline & Sig. (bilateral) & 0,872 & 0,214 & 0 & 0 & \\
\hline & $\mathrm{N}$ & 164 & 164 & 164 & 164 & 164 \\
\hline
\end{tabular}

**. La correlación es significativa al nivel 0,01 (bilateral).

*. La correlación es significante al nivel 0,05 (bilateral).

Con respecto al estilo de aprendizaje y los años de ingreso a la universidad, se observó que solo el estilo activo guarda relación con los años de ingreso (chi cuadrado $=31,197, p=0,002$ ). Por lo tanto, el año de ingreso de los estudiantes incide en el estilo de aprendizaje activo.

Al relacionar cada estilo de aprendizaje con el género de los alumnos, se visualiza que no existe asociación entre cada uno de los estilos de aprendizaje y la variable género $(p<0,05)$. Por lo tanto, se puede afirmar que las variables estilo de aprendizaje y género son independientes, en otras palabras, el género de los estudiantes no incide en los estilos de aprendizaje. Este resultado difiere de lo 
planteado por Alonso (2002), quien encontró que en los estilos teórico y activo existía una influencia del género.

Al comparar las medias para cada estilo de aprendizaje según rendimiento académico (Tabla 3), se observa que las categorías de rendimiento académico poseen igualdad de medias en los estilos activo, reflexivo y pragmático. De esa forma, no existen diferencias significativas en estos tres estilos. Por otro lado, con respecto al estilo teórico se evidencia que hay una diferencia significativa en el estilo teórico según el rendimiento académico de los estudiantes. Al respecto, los alumnos que obtuvieron mayores puntajes en el estilo teórico son aquellos categorizados con un rendimiento bueno (B), incluso un $61,54 \%$ de estos están ubicados dentro de las preferencias alta y muy alta para este estilo.

\section{Tabla 3}

Comparación de los estilos de aprendizaje con el rendimiento académico de los estudiantes de Psicología

\begin{tabular}{rrrrrrr}
\hline & & $\begin{array}{c}\text { Suma de } \\
\text { cuadrados }\end{array}$ & gl & $\begin{array}{r}\text { Media } \\
\text { cuadrática }\end{array}$ & F & Sig. \\
\hline Activo & Inter-grupos & 48,800 & 3 & 16,267 & 1,740 & 0,161 \\
\hline & Intra-grupos & 1496,048 & 160 & 9,350 & & \\
\hline Reflexivo & Total & 1544,848 & 163 & & & \\
\hline & Inter-grupos & 53,476 & 3 & 17,825 & 2,373 & 0,072 \\
\hline Intra-grupos & 1201,646 & 160 & 7,510 & & \\
\hline Teórico & Total & 1255,122 & 163 & & & \\
\hline & Inter-grupos & 71,605 & 3 & 23,868 & 2,690 & 0,048 \\
\hline Intra-grupos & 1419,639 & 160 & 8,873 & & \\
\hline Pragmático & Inter-grupos & 3,89 & 3 & 1,296 & 0,184 & 0,907 \\
\hline & Intra-grupos & 1129,06 & 160 & 7,057 & & \\
\hline Total & 1132,95 & 163 & & & \\
\hline
\end{tabular}


Al comparar las medias para cada estilo de aprendizaje según año de ingreso (Tabla 4), la muestra posee igualdad de medias en los estilos teórico y pragmático, es decir, no existen diferencias significativas entre estos dos estilos según los distintos años de ingreso de los estudiantes a la universidad. Por otro lado, hay una diferencia significativa en los estilos activo y reflexivo según el año de ingreso de los estudiantes. De esa forma, los alumnos que obtuvieron mayores puntajes en el estilo activo son aquellos que ingresaron a la universidad en el año 2007, ya que un $44,44 \%$ de estos están ubicados dentro de las preferencias alta y muy alta para este estilo. Por otro lado, el grupo que tuvo mayores puntajes en el estilo reflexivo, también fue el que ingresó en 2007 , pues un $38,70 \%$ de estos individuos están agrupado dentro de las preferencias alta y muy alta para este estilo.

\section{Tabla 4}

Comparación de los estilos de aprendizaje según el año de ingreso de los estudiantes de Psicología.

\begin{tabular}{rrrrrrr}
\hline & & $\begin{array}{c}\text { Suma de } \\
\text { cuadrados }\end{array}$ & gl & $\begin{array}{r}\text { Media } \\
\text { cuadrática }\end{array}$ & F & Sig. \\
\hline Activo & Inter-grupos & 125,362 & 3 & 41,787 & 4,710 & 0,004 \\
\hline Intra-grupos & 1419,486 & 160 & 8,872 & & \\
\hline Reflexivo & Inter-grupos & 1544,848 & 163 & & & \\
\hline & Intra-grupos & 1185,885 & 160 & 7,412 & & \\
\hline Teórico & Total & 1255,122 & 163 & & & \\
\hline & Inter-grupos & 27,457 & 3 & 9,152 & 1,000 & 0,394 \\
\hline Intra-grupos & 1463,787 & 160 & 9,149 & & \\
\hline Pragmático & Inter-grupos & 24,413 & 3 & 8,138 & 1,175 & 0,321 \\
\hline & Intra-grupos & 1108,539 & 160 & 6,928 & & \\
\hline Total & 1132,951 & 163 & & & \\
\hline
\end{tabular}


Al realizar una comparación de medias para cada estilo de aprendizaje según el sexo de los estudiantes (Tabla 5), se observó que la muestra posee igualdad de medias en los cuatro estilos de aprendizaje, por ello no visualizan diferencias significativas en los estilos de aprendizaje de los estudiantes según la variable género.

\section{Tabla 5}

Comparación de los estilos de aprendizaje según el sexo de los estudiantes de Psicología.

\begin{tabular}{rrrrrrr}
\hline & & $\begin{array}{c}\text { Suma de } \\
\text { cuadrados }\end{array}$ & gl & $\begin{array}{r}\text { Media } \\
\text { cuadrática }\end{array}$ & $F$ & Sig. \\
\hline Activo & Inter-grupos &, 443 & 1 &, 443 &, 046 &, 830 \\
\hline & Intra-grupos & 1544,405 & 162 & 9,533 & & \\
\hline Total & 1544,848 & 163 & & & \\
\hline & Inter-grupos &, 002 & 1 &, 002 &, 000 &, 987 \\
\hline Teórico & Intra-grupos & 1255,120 & 162 & 7,748 & & \\
\hline & Inter-grupos & 1255,122 & 163 & & & \\
\hline & Intra-grupos & 1489,395 & 162 & 9,194 & & \\
\hline Pragmático & Inter-grupos & 6,772 & 1 & 6,772 &, 974 &, 325 \\
\hline & Intra-grupos & 1126,179 & 162 & 6,952 & & \\
\hline Total & 1132,951 & 163 & & & \\
\hline & & 1491,244 & 163 & & & \\
\hline
\end{tabular}




\section{DISCUSIÓN}

El perfil de aprendizaje de la muestra, de acuerdo con las puntuaciones medias obtenidas en cada estilo de aprendizaje, evidenció preferencias moderadas para los cuatro estilos de aprendizaje medidos por el cuestionario de Honey-Alonso de Estilos de Aprendizaje (CHAEA).

De acuerdo con este resultado se puede concluir que, al momento de afrontar el proceso de aprendizaje, los estudiantes de la carrera de Psicología que participaron en el estudio presentan igual predominancia por: buscar nuevas experiencias y ser de mente abierta (estilo activo), anteponer la reflexión a la acción observando con detenimiento las distintas experiencias (estilo reflexivo), enfocar lógicamente los problemas integrando la experiencia en un marco teórico de referencia (estilo teórico) y, por último, aplicar ideas y aprovechar las oportunidades para experimentarlas (estilo pragmático). Por lo tanto, se podría inferir que los alumnos de la muestra tienen la capacidad de adecuarse a los distintos contextos del proceso de enseñanza-aprendizaje.

En cuanto a las preferencias por cada estilo de aprendizaje, es importante destacar que los mayores porcentajes se ubican en la preferencia moderada, es decir, que la mayor cantidad de alumnos prefiere de forma moderada cada uno de los estilos. Por otro lado, al sumar las preferencias más altas (alta y muy alta), para agrupar los mejores puntajes según los baremos de Honey y Alonso en cada estilo de aprendizaje de la muestra, se observó un mayor porcentaje en los estilos teórico y activo, seguido por el pragmático. El estilo reflexivo es el que presentó el menor porcentaje de preferencia alta y muy alta.

Lo anterior, se relaciona con la teoría expuesta por Alonso, Gallego \& Honey (2002), quienes sostienen que, al poseer preferencias altas y muy altas en el estilo activo, el aprendizaje se verá facilitado cuando se esté expuesto a un método de enseñanza en el cual: se intentan cosas nuevas, se representan roles, se generan ideas sin limitaciones formales, se presentan oportunidades de dirigir debates, resolver problemas siendo parte de un equipo, entre otros. En el caso de altas y muy altas preferencias en el estilo teórico, el aprendizaje se verá facilitado cuando: se tenga tiempo para explorar metódicamente las asociaciones y relaciones entre ideas, se cuente con la posibilidad de cuestionar, se logre poner a prueba métodos y lógica que sean la base de algo, se participe en situaciones complejas, entre otros. 
Además, no se encontraron correlaciones estadísticamente significativas entre rendimiento académico y los estilos reflexivo, teórico y pragmático. Por su parte, el estilo activo se correlacionó negativamente con el rendimiento, lo que indicó que los estudiantes con mayor rendimiento académico presentan menores puntajes en el estilo activo. Visto de otra forma, el ser animador, improvisador, arriesgado, espontáneo, vivir en búsqueda de experiencias nuevas, entre otras, son características que afectaron negativamente el rendimiento académico de los alumnos. Esto podría indicar que las características anteriormente señaladas no serían las más esenciales en el proceso de aprendizaje de los estudiantes de Psicología de una universidad de la ciudad de Talca en Chile.

Según lo expuesto, se podría inferir que existen otros factores, además de los estilos de aprendizaje, que también influyen en el rendimiento académico de los alumnos de la muestra. Suazo (2007) expone que estos factores han sido agrupados en cuatro grandes categorías: las características de la institución educacional (abarca dirección, gestión, recursos, infraestructura, clima institucional); aspectos curriculares en los que se desarrollan los ramos (engloba sistemas didácticos, programas, carga académica); los profesores de las asignaturas (género, edad, grado de preparación, interacción con el estudiante) y estudiante (involucra variables como las demográficas, familiares, psicológicas y académicas).

Al comparar las medias de los estilos de aprendizaje según el rendimiento académico, se evidenció diferencias significativas solo en el estilo de aprendizaje teórico. Esto, lo que quiere decir, es que se presentan diferencias en este estilo según las distintas categorías en las que se estratificó el rendimiento académico. Los alumnos que obtuvieron mayores puntajes (preferencia alta y muy alta) en el estilo teórico son aquellos categorizados con un rendimiento bueno (B) y los que tuvieron puntajes menores (preferencia baja y muy baja) son aquellos categorizados con un rendimiento suficiente (S).

Con respecto al año de ingreso de los estudiantes, se observó que solo el estilo activo correlacionó significativamente con esta variable, lo que implica que el año de ingreso de los alumnos incide en el estilo de aprendizaje activo de estos. Luego, al analizar la existencia de diferencias significativas entre las medias de estas mismas variables, el estilo activo volvió a tomar relevancia, pues se observaron diferencias significativas entre este estilo y el reflexivo, lo que indicó que los puntajes obtenidos por los estudiantes en estos estilos varían según los distintos años de ingreso a la universidad. De esa forma, en el estilo activo y reflexivo, los que obtuvieron mayores puntajes (preferencias alta y muy alta) 
fueron los estudiantes que ingresaron a la universidad en el año 2007. Por otro lado, los menores puntajes (preferencia baja y muy baja) en el estilo activo fueron de los alumnos con año de ingreso 2006 y en el estilo reflexivo quienes entraron a la universidad en el 2004.

Los resultados anteriores indican que los alumnos con menos tiempo en la carrera de Psicología (año de ingreso 2007) tienden a ser más animadores, improvisadores, arriesgados, creativos y lideres, como también, ponderados, minuciosos, observadores, analíticos y pacientes. Esto se podría deber al hecho de que los estudiantes están entrando a un nuevo proceso en el que se deben asumir nuevas responsabilidades y responder de forma eficiente a las tareas académicas propuestas por un nivel de enseñanza más exigente, en el que se hace necesario entrar con energía e iniciativa, reflexionando mucho más para así lograr el aprendizaje esperado.

En cuanto al género de los estudiantes, se puede indicar que esta variable no incide en los estilos de aprendizaje de la muestra estudiada, por lo tanto, se concluye que las variables estilos de aprendizaje y género son independiente entre ellas en este estudio.

Con respecto a la comparación de medias de los estilos de aprendizaje según el género de los estudiantes, se evidenció que la muestra no posee diferencias significativas en cuanto a sus medias en cada estilo de aprendizaje.

Los resultados obtenidos en el estudio entregan una visión alentadora en cuanto a las preferencias de estilos de aprendizaje de los estudiantes de la carrera de Psicología, pues un perfil de aprendizaje que evidencia preferencias moderadas en cada estilo es una buena base para comenzar a potenciar los estilos de aprendizaje, pudiendo optar a las preferencias más altas de los baremos expuestos en esta investigación. No obstante, no hay que olvidar que existe un grupo importante de alumnos que ya se encuentran dentro de las preferencias alta y muy alta, los cuales, si desean favorecer su aprendizaje, pueden acceder a las bases teóricas propuestas por Alonso, Gallego y Honey, las que explican los aspectos que benefician el aprendizaje cuando se posee estas preferencias.

Finalmente, para fortalecer el aprendizaje de los estudiantes, se cree que, en primera instancia, los docentes deberían realizar un autodiagnóstico de sus estilos de aprendizaje predominantes, ya que a partir de su propia experiencia podrían ayudar a los alumnos a entender mejor sus estilos de aprendizaje, permitiendo así 
una mejor y más acertada utilización de recursos en el proceso de enseñanzaaprendizaje.

Otro punto importante, sería relacionar los estilos de aprendizaje con los estilos de enseñanza de los docentes, pues el establecer la relación entre la forma de aprender y de enseñar convierte la experiencia educativa en una práctica satisfactoria tanto para los docentes como para los estudiantes. Indudablemente, es necesario, que los docentes sean capaces de desarrollar un repertorio de estrategias de enseñanza eficaces de manera espontánea, mientras cada aprendiz avanza en su aprendizaje, dado que los resultados exitosos requieren de docentes gestores de cambio y de calidad.

Por otro lado, sería importante crear un plan de acción para fomentar los estilos de aprendizaje que se presentan más fuertes y débiles en los estudiantes. Ese proyecto debe valorar tanto las formas preferidas como las menos preferidas de los alumnos para aprender; también, debe incluir una variedad de métodos, procedimientos de enseñanza y aprendizaje, medios y formas de evaluar, que propicien el interés, la participación e implicación de los estudiantes en las tareas de aprendizaje, desarrollando sus fortalezas y debilidades.

\section{REFERENCIAS BIBLIOGRÁFICAS}

ALONSO, C., GALLEGO, D. \& HONEY, P. (2002). Los Estilos de Aprendizaje. Bilbao: Ediciones Mensajero.

CABRERA, J. \& FARIÑAS, G. (2005). El estudio de los estilos de aprendizaje desde una perspectiva vigostkiana: una aproximación conceptual. Extraído el 15 de junio de 2007 desde http://www.rieoei.org/deloslectores/1090Cabrera.pdf

DELGADO, A. (2004). Relación entre los estilos de aprendizaje y los estilos de pensamiento en estudiantes de maestría considerando las especialidades profesionales y el tipo de universidad. Extraído el 15 de noviembre de 2006 desde http://www.cybertesis.edu.pe/sisbib/2004/delgado_va/html/index-frames.html

GÓMEZ, L., ADUNA, M., GARCÍA, E., CISNEROS, A. \& PADILLA, J. (2004). Manual de Estilos de Aprendizaje. Extraído el 1 de marzo de 2007 desde http:// www.dgb.sep.gob.mx/servedu/docentes/actualizacion/multimedia/Manual.pdf 
LÓPEZ, C. \& BALLESTEROS, B. (2003). Evaluación de los estilos de aprendizaje en estudiantes de enfermería mediante el cuestionario CHAEA. Enfermería Global: Revista electrónica mensual de enfermería. 3. Extraído el 5 de mayo de 2007 desde http://dialnet.unirioja.es/servlet/articulo?codigo=763802

MARTínEZ, R. (2003). Los estilos de aprendizaje de los estudiantes de Administración de Hoteles y Restaurantes y profesionales hoteleros. Extraído el 15 de julio de 2007 desde http://catarina.udlap.mx/u_dl_a/tales/documentos/hr/ martinez_c_rl

NAVARRO, M. (2001). Estilos de aprendizaje en alumnos de primaria. Revista de Filología y su didáctica. 24, 565-582. Extraído el 8 de noviembre de 2007 desde http://www.institucional.us.es/revistas/revistas/cauce/pdf/numeros/24/Navarro.pdf

RODRÍGUEZ, M. (2003). Pautas para reducir los estereotipos sexistas en orientación profesional. Una propuesta metodológica. Extraído el 6 de mayo de 2007 desde http://web.educastur.princast.es/proyectos/coeduca/wp-content/ uploads/2006/08/pautas.pdf

ROMO, M., LÓPEZ, D., TOVAR, J. Y LÓPEZ, I. (2004). Estilos de aprendizaje de estudiantes de nutrición y dietética. Revista Praxis. 5, 9-24. Extraído el 20 de marzo de 2007 desde http://revistapraxis.cl/ediciones/numero5/romo_praxis5.pdf

RUIZ, B., TRILLOS, J. \& MORALES, J. (2006). Estilos de aprendizaje y rendimiento académico en estudiantes universitarios. Revista de estudios e investigación en psicología y educación, 13, 441-460. Extraído el 15 de junio de 2007 desde http:// dialnet.unirioja.es/servlet/articulo?codigo $=2138907$

SAAVEDRA, E. \& REYNALDOS, C. (2006). Caracterización cognitiva y emocional de los estudiantes de la Universidad Católica del Maule. Estudios Pedagógicos, 32 (2), 87-102.

SANDÍN, M. (2003). Investigación cualitativa en educación. Fundamentos y tradiciones. Madrid: Mc Graw Hill.

SUAZO, I. (2007). Estilos de aprendizaje y su correlación con el rendimiento académico en anatomía humana normal. International Journal of Morphology. 25, 367-373. Extraído el 21 de abril de 2007 desde http://www.scielo.cl/scielo.php?pid $=$ S071795022007000200022\&script=sci_arttext 
THOMPSON, S. \& AVELEYRA, E. (2003). Estilos de Aprendizaje en Matemática. Extraído el 10 de marzo de 2007 desde http://www.fceco.uner.edu.ar/cpn/catedras/ matem1/educmat/em22ta.doc

VON CHRISMAR, A. (2005). Identificación de los estilos de aprendizaje y propuesta de orientación pedagógica para estudiantes de la Universidad Austral de Chile. Tesis para optar al grado de magíster en Modelado del conocimiento para entornos educativos virtuales, Facultad de Ciencias de la Ingeniería, Universidad Austral de Chile.

WOOLFOLK, A. (2015). Psicología Educativa. México: Pearson. 